\title{
Editorial
}

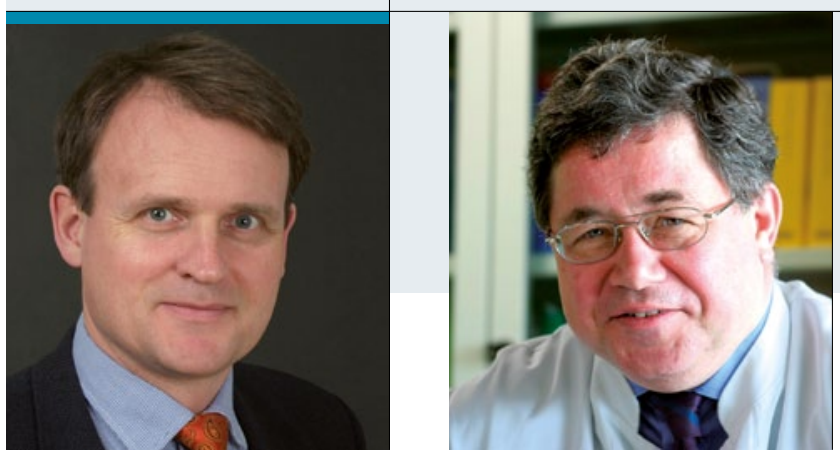

"Das Allergo Journal wird dieses Jahr

20 Jahre alt - ein willkommener Anlass für

eine gründliche Verjüngungskur."

Prof. Dr. Thilo Jakob, Allergieabteilung \& Forschergruppe Allergologie, Universitäts-Hautklinik Freiburg Prof. Dr. Hans F. Merk, Hautklinik der Medizinischen Fakultät, Universitätsklinikum der RWTH Aachen

\section{Allergo Journal 2.0}

Z um Jahresbeginn 2012 präsentieren wir Ihnen das Allergo Journal in neuem Gewand. Ein willkommener Anlass für diese Verjüngungskur ist das 20-jährige Jubiläum des Allergo Journals.

Das erste und wichtigste Ziel der Überarbeitung ist es, die Attraktivität für unsere Leser weiter zu erhöhen. Wie Sie sehen, hat sich nicht nur das äußere Erscheinungsbild geändert, sondern auch die Strukturierung des Inhaltes. Hierbei haben wir Altbewährtes beibehalten und um einige Innovationen bereichert: Zur besseren Übersichtlichkeit werden die begutachteten wissenschaftlichen Beiträge (Originalbeiträge, Übersichtsarbeiten, Fallberichte und Leitlinien) klar getrennt von den journalistisch aufbereiteten Rahmenartikeln, die Ihnen - quasi als "Reader's Digest" - in gewohnter Weise neueste Nachrichten aus Grundlagenforschung, klinischer Forschung und der Politik bieten werden. Ein klares Layout und ein Farbstreifen am Kopf der Seiten unterstützen diese Unterteilung. Zusätzlich informieren wir Sie unter „ÄDA/DGAKI informieren“ über Aktuelles und Mitteilungen aus den beiden allergologischen Verbänden.

Denjenigen von Ihnen, die auch das Kleingedruckte lesen, wird aufgefallen sein, dass wir den Bereich der klinischen Immunologie in unser Themenspektrum aufgenommen haben. Hier sollen vor allem die Bereiche der klinischen Immunologie mit direktem Bezug zur Allergologie abgedeckt werden - sei es aufgrund differenzialdiagnostischer Relevanz oder aufgrund der Ähnlichkeit der beteiligten immunologischen Mechanismen. Besonders im Bereich der Immundefizienz-Forschung und auf dem Gebiet der autoinflammatorischen Erkrankungen gibt es viele neue Erkenntnisse, die für das Verständnis der klinischen Allergologie von Bedeutung sein werden. Mit der ersten Ausgabe 2012 firmiert das Allergo Journal somit nun unter dem offiziellen Namen „Interdisziplinäre Zeitschrift für Allergologie, klinische Immunologie und Umweltmedizin“.

Was haben wir uns noch vorgenommen für das Jahr 2012? Die große Resonanz auf die CME-Beiträge der Vergangenheit hat uns dazu veranlasst, künftig möglichst in jedem Heft eine zertifizierte Fortbildung an- zubieten. Die Im-Fokus-Beitragsserie zur molekularen Allergologie wurde ebenfalls sehr gut angenommen und wird 2012 fortgesetzt. In Anlehnung an diese Beiträge wird aktuell von Mitgliedern der Sektion Immunologie der DGAKI eine neue Serie vorbereitet: „Das 1x1 der Immunologie für den praktizierenden Allergologen“. Hier sollen neue Entwicklungen der Immunologie in kurzer, prägnanter und verständlicher Weise dargestellt werden. $\mathrm{Zu}$ guter Letzt haben wir noch eine Serie zu Themen der Rhinologie geplant.

Wir hoffen mit diesen Änderungen und Innovationen Ihre Bedürfnisse nach fundierter, aktueller allergologischer Fortbildung zu treffen. Sollten Sie Anregungen oder Themenwünsche haben, möchten wir Sie herzlich einladen, uns dies mitzuteilen.

Ein weiteres wichtiges Thema ist die Erreichbarkeit und Sichtbarkeit der Beiträge im Allergo Journal. Hier haben wir bereits umgesetzt, dass alle Inhalte über www.springermedizin.de/allergo-journal verfügbar sind. Zusätzlich werden die wissenschaftlichen Beiträge im Laufe des Jahres auch in das Online-Portal Springerlink (www.springerlink.com) eingestellt werden und somit über die meisten institutionellen elektronischen Bibliotheken verfügbar sein. Außerdem wählen die Herausgeber künftig in jeder Ausgabe mindestens einen Artikel aus, der zusätzlich ins Englische übersetzt und online frei verfügbar sein wird. Hiermit sollte es uns gelingen, dem offiziellen Organ der DGAKI und des ÄDA international eine größere Sichtbarkeit zu verschaffen.

Wir sind selbst gespannt, wie sich all unsere guten Vorsätze umsetzen lassen und auf welche Resonanz sie stoßen werden. Wir freuen uns, mit Ihnen zusammen die Attraktivität des Allergo Journals weiter zu optimieren.

Viel Freude bei der Lektüre der aktuellen Ausgabe und beste Wünsche für ein glückliches und erfolgreiches 2012 .

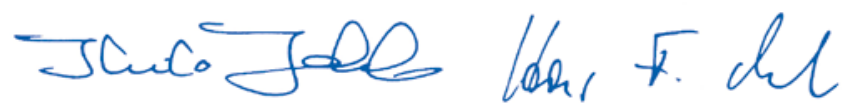

